Pacific Journal of Mathematic 


\title{
A REPRESENTATION FOR THE LOGARITHMIC DERIVATIVE OF A MEROMORPHIC FUNCTION
}

\author{
MORRIS MARDEN
}

\begin{abstract}
A new representation is developed for the logarithmic derivative of a meromorphic function $f$ in terms of its zeros and poles, using as parameters some of the critical points of $f$. Applications are made to locating all but a finite number of critical points of $f$.
\end{abstract}

\section{The principal result.}

THEOREM 1.1. Let $f$ be a meromorphic function of finite order $\rho$ possessing the finite zeros $a_{1}, a_{2}, a_{3}, \cdots$ and poles $b_{1}, b_{2}, b_{3}, \cdots$. Let $\zeta_{1}, \zeta_{2}, \ldots \zeta_{n}$, be any $n=[\rho]$ distinct zeros of the derivative $f^{\prime}$ of $f$ which are not also zeros of $f$. Then for $z \neq a_{j}, b_{j}(j=1,2,3, \cdots)$

$$
\frac{f^{\prime}(z)}{f(z)}=\sum_{j=1}^{\infty} \frac{\psi(z)}{\psi\left(a_{j}\right)\left(z-a_{j}\right)}-\sum_{j=1}^{\infty} \frac{\psi(z)}{\psi\left(b_{j}\right)\left(z-b_{j}\right)}
$$

where $\psi(z)=1$ for $n=0$,

$$
\psi(z)=\left(z-\zeta_{1}\right)\left(z-\zeta_{2}\right) \cdots\left(z-\zeta_{n}\right) \quad \text { for } n>0 .
$$

In (1.1) the convergence is uniform on every compact set excluding all the $a_{j}$ and $b_{j}$.

In the case that $f$ is a rational function with $m$ zeros and $p$ poles, identity (1.1) reduces to the familiar formula

$$
f^{\prime}(z) / f(z)=\sum_{j=1}^{m}\left(z-a_{j}\right)^{-1}-\sum_{j=1}^{p}\left(z-b_{j}\right)^{-1} .
$$

Furthermore, if the second summation is omitted in (1.1), identity (1.1) reduces to one which we had previously obtained [See 1] for entire functions of finite order.

2. Proof. Being a meromorphic function, $f$ can be written as a ratio of two entire functions, each of which has an Hadamard representation in terms of its zeros. Thus,

$$
f(z)=z^{m} e^{P(z)} \prod_{j=1}^{\infty}\left[E\left(z / a_{j}, p\right) / E\left(z / b_{j}, q\right)\right]
$$

where $m$ is an integer (positive, negative or zero); $P(z)$ is a polynomial of degree at most $n=[\rho] ; p$ and $q$ are nonnegative integers not exceeding $n$ and 


$$
E(u, p)=(1-u) \exp \left[u+(1 / 2) u^{2}+\cdots+(1 / p) u^{p}\right]
$$

if $p>0$ whereas $E(u, 0)=(1-u)$. Taking the logarithmic derivative of (2.1) and simplifying, one finds that

$$
\frac{f^{\prime}(z)}{f(z)}=\frac{m}{z}+P^{\prime}(z)+A(z)-B(z)
$$

where

$$
A(z)=\sum_{\jmath=1}^{\infty} \frac{z^{p}}{a_{\jmath}^{p}\left(z-a_{j}\right)}, B(z)=\sum_{\jmath=1}^{\infty} \frac{z^{q}}{b_{\jmath}^{q}\left(z-b_{\jmath}\right)} .
$$

By hypothesis, $f^{\prime}\left(\zeta_{k}\right)=0, k=1,2, \cdots, n$. Hence, from (2.2), follows that for $k=1,2, \cdots, n$

$$
P^{\prime}\left(\zeta_{k}\right)=-\left(m / \zeta_{k}\right)-A\left(\zeta_{k}\right)+B\left(\zeta_{k}\right) .
$$

Since $P^{\prime}(z)$ is a polynomial of degree at most $n-1$, it can be represented by the Lagrange Interpolation Formula as

$$
\frac{P^{\prime}(z)}{\psi^{\prime}(z)}=\sum_{k=1}^{n} \frac{P^{\prime}\left(\zeta_{k}\right)}{\psi^{\prime}\left(\zeta_{k}\right)\left(z-\zeta_{k}\right)}
$$

Hence, using (2.3) and (2.4), one finds that

$$
\begin{aligned}
\frac{P^{\prime}(z)}{\psi(z)}= & -\sum_{k=1}^{n} \frac{m}{\zeta_{k} \psi^{\prime}\left(\zeta_{k}\right)\left(z-\zeta_{k}\right)}-\sum_{k=1}^{n} \sum_{j=1}^{\infty} \frac{\zeta_{k}^{p}}{a_{\jmath}^{p} \psi^{\prime}\left(\zeta_{k}\right)\left(z-\zeta_{k}\right)\left(\zeta_{k}-a_{j}\right)} \\
& +\sum_{k=1}^{n} \sum_{j=1}^{\infty} \frac{\zeta_{k}^{q}}{b_{J}^{q} \psi^{\prime}\left(\zeta_{k}\right)\left(z-\zeta_{k}\right)\left(\zeta_{k}-b_{j}\right)} .
\end{aligned}
$$

In view of the fact that sums $A(z)$ and $B(z)$ are uniformly and absolutely convergent on every compact set that omits all the $a_{j}$ and $b_{j}$, the order of summation of the double sums in (2.5) can be reversed. Thus the first double sum in (2.5) becomes

$$
\sum_{j=1}^{\infty} \frac{1}{a_{\jmath}^{p}} \sum_{k=1}^{n} \frac{\zeta_{k}^{p}}{\psi^{\prime}\left(\zeta_{k}\right)\left(\zeta_{k}-a_{j}\right)\left(z-\zeta_{k}\right)}=\sum_{j=1}^{\infty} \frac{1}{a_{\jmath}^{p}\left(z-a_{j}\right)}\left[\frac{S(z)}{\psi(z)}-\frac{S\left(a_{j}\right)}{\psi\left(a_{j}\right)}\right]
$$

where

$$
S(z)=\psi(z) \sum_{k=1}^{n} \frac{\zeta_{k}^{p}}{\left(z-\zeta_{k}\right) \psi^{\prime}\left(\zeta_{k}\right)}
$$

Since the polynomial $S(z)$ is of degree at most $n-1$ with $S\left(\zeta_{k}\right)=\zeta_{k}^{p}$, the polynomial

$$
T(z)=S(z)-z^{p}
$$

is of degree at most $n$ such that 


$$
T\left(\zeta_{k}\right)=0, \text { for } k=1,2, \cdots, n .
$$

Therefore $T(z)=c \psi(z)$, where $c$ is a constant that may be zero. Accordingly,

$$
S(z)=z^{p}+c \psi(z)
$$

and the sum (2.6) becomes

$$
\sum_{j=1}^{\infty} \frac{z^{p}}{\psi(z) a_{\jmath}^{p}\left(z-a_{j}\right)}-\sum_{j=1}^{\infty} \frac{1}{\psi\left(a_{j}\right)\left(z-a_{j}\right)} .
$$

Similarly the second double sum in (2.5) reduces to

$$
\sum_{j=1}^{\infty} \frac{z^{q}}{\psi(z) b_{j}^{p}\left(z-b_{j}\right)}-\sum_{j=1}^{\infty} \frac{1}{\psi\left(b_{j}\right)\left(z-b_{j}\right)} .
$$

Finally, on use of the Lagrange Interpolation Formula for $1 / \psi(z)$, the single sum in (2.5) becomes

$$
\frac{m}{z} \sum_{k=1}^{n}\left[\frac{1}{\zeta_{k}}+\frac{1}{z-\zeta_{k}}\right] \frac{1}{\psi^{\prime}\left(\zeta_{k}\right)}=\frac{m}{z}\left[-\frac{1}{\psi(0)}+\frac{1}{\psi(z)}\right] .
$$

Substituting from (2.9), (2.10) and (2.11) into (2.5), one reduces (2.2) to

$$
\frac{f^{\prime}(z)}{f(z)}=\frac{m \psi(z)}{z \psi(0)}+\sum_{j=1}^{\infty} \frac{\psi^{\prime}(z)}{\psi\left(a_{j}\right)\left(z-a_{j}\right)}-\sum_{j=1}^{\infty} \frac{\psi(z)}{\psi\left(b_{j}\right)\left(z-b_{j}\right)} .
$$

However, the first term here may be dropped since it is obtainable from the first or second sum in (2.12) by allowing either $m a_{j}$ (if $m>0$ ) or $-m b_{j}$ (if $m<0$ ) to coalesce at 0 . Thus identity (1.1) has been established.

3. Location of critical points. An immediate consequence of Theorem 1.1 is the following:

THEOREM 3.1. Let $f$ be a meromorphic function of finite order $\rho$ possessing the finite zeros $a_{1}, a_{2}, a_{3}, \cdots$ and poles $b_{1}, b_{2}, b_{3}, \cdots$ and let $\zeta_{0}, \zeta_{1}, \cdots, \zeta_{n}$, be any $n+1=[\rho]+1$ distinct critical points of $f$ which are not also zeros of $f$. Then

$$
\sum_{j=1}^{\infty} \frac{1}{\left(\zeta_{0}-a_{j}\right)\left(\zeta_{1}-a_{j}\right) \cdots\left(\zeta_{n}-a_{j}\right)}=\sum_{j=1}^{\infty} \frac{1}{\left(\zeta_{0}-b_{j}\right)\left(\zeta_{1}-b_{j}\right) \cdots\left(\zeta_{n}-b_{j}\right)} .
$$

Equation (3.1) follows from (1.1) on setting $z=\zeta_{0}$, writing out $\psi\left(a_{j}\right)$ according to (1.2) and cancelling the factor $\psi\left(\zeta_{0}\right) \neq 0$.

As an application of (3.1), the following will now be proved. 
THeOREm 3.2. Let $D_{a}$ and $D_{b}$ be two regions with which can be associated a set $R$ of points $\zeta$ such that a ray from $\zeta$ to some point $\gamma$ separates $\bar{D}_{a}$ from $\bar{D}_{b}$ and such that inequality

$$
0<\arg [(\gamma-\zeta) /(z-\zeta)]<\pi /(n+1)
$$

holds for all $z$ in one of the regions $D_{a}, D_{b}$ and inequality

$$
-\pi /(n+1)<\arg [\gamma-\zeta) /(z-\zeta)]<0
$$

holds for all $z$ in the other region. Let $f$, a meromorphic function of finite order $\rho$, have all its zeros in $D_{a}$ and all its poles in $D_{b}$. Then at most $n=[\rho]$ critical points of $f$ lie in $R$.

Proof. If on the contrary $n+1$ distinct critical points $\zeta_{0}, \zeta_{1}, \cdots, \zeta_{n}$ were in $R$, identity (3.1) holds for them in relation to the zeros and poles of $f$. By hypothesis, one can associate with each $\zeta_{k}$, a point $\gamma_{k}$ such that for $j=1,2,3, \cdots$ the inequalities

$$
\begin{gathered}
0<\arg \frac{\gamma_{k}-\zeta_{k}}{a_{j}-\zeta_{k}}<\frac{\pi}{n+1} \\
-\frac{\pi}{n+1}<\arg \frac{\gamma_{k}-\zeta_{k}}{b_{j}-\zeta_{k}}<0
\end{gathered}
$$

hold (or those with $a_{j}$ and $b_{j}$ interchanged).

Setting

$$
T(z)=\prod_{k=0}^{n}\left[\left(\gamma_{k}-\zeta_{k}\right) /\left(z-\zeta_{k}\right)\right]
$$

one infers that

$$
0<\arg T\left(a_{j}\right)<\pi, \quad-\pi<\arg T\left(b_{j}\right)<0,
$$

for all $j$. This means that

$$
\begin{array}{r}
0<\arg \sum_{j=1}^{\infty} T\left(a_{j}\right)<\pi, \\
-\pi<\arg \sum_{j=1}^{\infty} T\left(b_{j}\right)<0 .
\end{array}
$$

Consequently,

$$
\sum_{j=1}^{\infty} T\left(a_{j}\right) \neq \sum_{j=1}^{\infty} T\left(b_{j}\right)
$$

in contradiction to (3.1). Consequently, at most $n$ distinct critical points $\zeta_{k}$ can lie on $R$, as was to be proved.

As an illustration, let $f$ be a meromorphic function of order $\rho$, 
$1 \leqq \rho<\infty$, and let

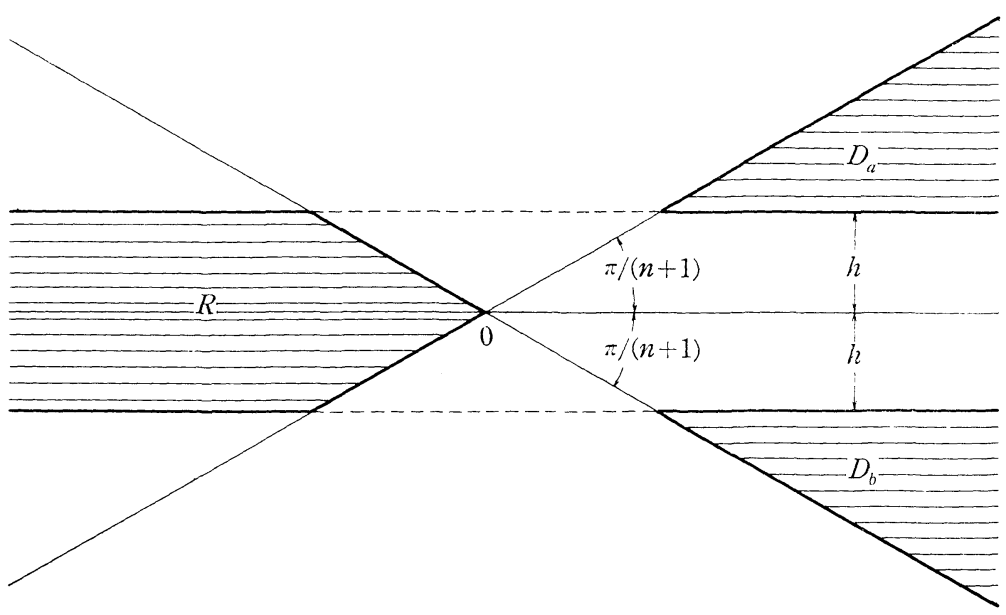

Figure 1

$$
\begin{aligned}
& D_{a}=\{z=x+i y: x>0, \quad 0 \leqq h<y<x \tan [\pi /(n+1)]\} \\
& D_{b}=\{z=x+i y: x>0, \quad-h>y>-x \tan [\pi /(n+1)]\} \text {. }
\end{aligned}
$$

Then, according to Theorem 3.2, at most $n$ critical points of $f$ lie in the region

$$
R=\{z=x+i y: x<0, \quad|y|<\min [h,|x| \tan [\pi /(n+1)]\}
$$

REMARK. In identity (1.1) and the subsequent theorems, $f^{\prime}(z)$ may be replaced by the linear combination $f^{\prime}(z)+\lambda f(z)$ or more generally by

$$
F_{1}(z)=f^{\prime}(z)+f(z) g^{\prime}(z)
$$

where $g(z)$ is an arbitrary polynomial of degree at most $n$, provided $\zeta_{0}, \zeta_{1}, \zeta_{2}, \cdots \zeta_{n}$ are taken as the zeros of $F_{1}(z)$. This follows from the fact that the meromorphic function $F(z)=e^{g(z)} f(z)$ is also of order $\rho$, has the same zeros and poles as $f$ and $F^{\prime}(z)=e^{g(z)} F_{1}(z)$.

\section{REFERENCE}

1. M. Marden, Logarithmic derivative of entire function, Proc. Amer. Math. Soc., 27 (1971), 513-518.

Received October 23, 1970. Partially supported by NSF Grant No. GP-19615. Presented by title to American Mathematical Society, abstract.

UNIVERSITY OF WISCONSIN-MILWAUKEE 



\section{PACIFIC JOURNAL OF MATHEMATICS}

\section{EDITORS}

H. SAMELSON

Stanford University

Stanford, California 94305

C. R. Hовву

University of Washington

Seattle, Washington 98105
J. DUGUNDJI

Department of Mathematics

University of Southern California

Los Angeles, California 90007

RICHARD ARENS

University of California

Los Angeles, California 90024

\section{ASSOCIATE EDITORS}
E. F. BECKENBACH
B. H. NeumanN
F. WOLF
K. YoSHIDA

\section{SUPPORTING INSTITUTIONS}

UNIVERSITY OF BRITISH COLUMBIA

CALIFORNIA INSTITUTE OF TECHNOLOGY

UNIVERSITY OF CALIFORNIA

MONTANA STATE UNIVERSITY

UNIVERSITY OF NEVADA

NEW MEXICO STATE UNIVERSITY

OREGON STATE UNIVERSITY

UNIVERSITY OF OREGON

OSAKA UNIVERSITY

UNIVERSITY OF SOUTHERN CALIFORNIA
STANFORD UNIVERSITY

UNIVERSITY OF TOKYO

UNIVERSITY OF UTAH

WASHINGTON STATE UNIVERSITY

UNIVERSITY OF WASHINGTON

AMERICAN MATHEMATICAL SOCIETY CHEVRON RESEARCH CORPORATION NAVAL WEAPONS CENTER 


\section{Pacific Journal of Mathematics}

\section{Vol. 38, No. $1 \quad$ March, 1971}

Bruce Alan Barnes, Banach algebras which are ideals in a Banach algebra ..... 1

David W. Boyd, Inequalities for positive integral operators............... 9

Lawrence Gerald Brown, Note on the open mapping theorem .............. 25

Stephen Daniel Comer, Representations by algebras of sections over Boolean

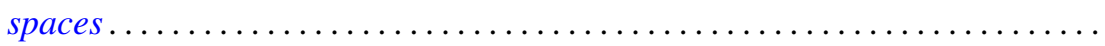

John R. Edwards and Stanley G. Wayment, On the nonequivalence of

conservative Hausdorff methods and Hausdorff moment sequences ........

P. D. T. A. Elliott, On the limiting distribution of additive functions $(\bmod 1) \ldots \ldots$

Mary Rodriguez Embry, Classifying special operators by means of subsets

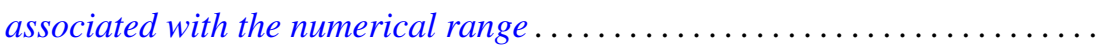

Darald Joe Hartfiel, Counterexamples to a conjecture of G. N. de Oliveira ......

C. Ward Henson, A family of countable homogeneous graphs...............

Satoru Igari and Shigehiko Kuratsubo, A sufficient condition for

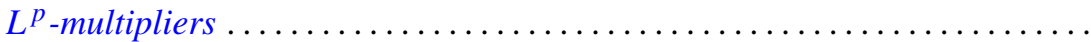

William A. Kirk, Fixed point theorems for nonlinear nonexpansive and

generalized contraction mappings............................

Erwin Kleinfeld, A generalization of commutative and associative rings ...... 95

D. B. Lahiri, Some restricted partition functions. Congruences modulo $11 \ldots \ldots 103$

T. Y. Lin, Homological algebra of stable homotopy ring $\pi *$ of spheres ....... 117

Morris Marden, A representation for the logarithmic derivative of a meromorphic function...........................

John Charles Nichols and James C. Smith, Examples concerning sum properties for metric-dependent dimension functions . .

Asit Baran Raha, On completely Hausdorff-completion of a completely

Hausdorff space.

M. Rajagopalan and Bertram Manuel Schreiber, Ergodic automorphisms and affine transformations of locally compact groups..........

N. V. Rao and Ashoke Kumar Roy, Linear isometries of some function

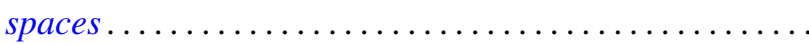

William Francis Reynolds, Blocks and F-class algebras of finite groups

Richard Rochberg, Which linear maps of the disk algebra are multiplicative ...

Gary Sampson, Sharp estimates of convolution transforms in terms of decreasing

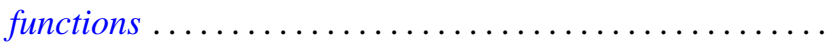

Stephen Scheinberg, Fatou's lemma in normed linear spaces

Ken Shaw, Whittaker constants for entire functions of several complex

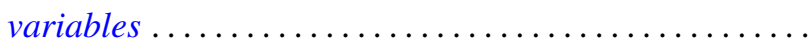

James DeWitt Stein, Two uniform boundedness theorems................ 251

$\mathrm{Li} \mathrm{Pi} \mathrm{Su,} \mathrm{Homomorphisms} \mathrm{of} \mathrm{near-rings} \mathrm{of} \mathrm{continuous} \mathrm{functions} \mathrm{.} \mathrm{.............} 261$

Stephen Willard, Functionally compact spaces, $C$-compact spaces and mappings of minimal Hausdorff spaces....................... 